EPJ Web of Conferences 45, 01113 (2013)

DOI: $10.1051 /$ epjconf/20134501113

(C) Owned by the authors, published by EDP Sciences, 2013

\title{
The influence of the sleeve's orifices geometric patterns on the fluid flow through a hydraulic resistance
}

\author{
I. Sfarlea ${ }^{1}$, F. Bode ${ }^{1 \mathrm{a}}$ and D. Opruta ${ }^{1}$ \\ ${ }^{1}$ Technical University of Cluj Napoca, Faculty of Mechanics, Dept. of Mechanical Engeneering, Romania
}

\begin{abstract}
The present paper provides a CFD (Computational Fluid Dynamics) type analysis on a fluid flow through an hydraulic resistance with cylindrical slide and 4 orifices sleeve. Its purpose is to determine the optimal geometric configuration for this type of hydraulic resistances, in order to enhance the dynamic characteristics of control devices. The issues under study were velocity fiel's distribution, aiming the velocity vectors orientation angle in the narrowest area, the flow regime, by determining the Reynolds number's numerical value and the pressure field's distribution, the analysis showing a sharp pressure drop due to the flow sections's variation. Also, given the sudden pressure drop, it was necessary to highlight the areas where cavitation may occur.
\end{abstract}

\section{Introduction}

The present paper deals with a numerical CFD simulation on a hydraulic resistance with cylindrical spool and sleeve with circular orifices. This study is part of a bigger project that regards the influence of geometric patterns of the sleeve's orifices on the fluid flow through a hydraulic resistance.

The aim of this study is the interpretation of the phenomena occurring during flow, in terms of analyzing the velocity and pressure fields' distribution.

Literature [1-4] provides more information on the sleeveless hydraulic resistances than for the one with sleeve. The difference between them is that the latter offers the possibility of a better flow control by having the same flow section, but with higher values for the spools' command openings, as shown in the flow equation through a diaphragmatic resistance (figure 1):

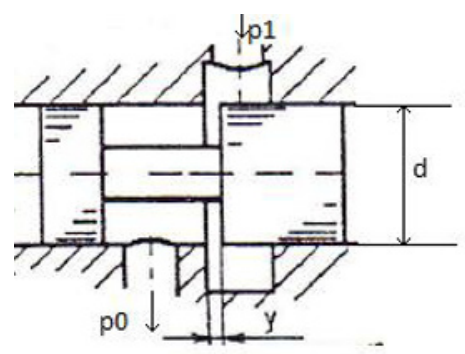

Fig.1 Diaphragmatic resistance

$$
Q=\alpha A \sqrt{\frac{2 \Delta p}{\rho}}(1),
$$

where

Q - fluid flow

A - flow section

$\Delta \mathrm{p}$ - pressure drop

$\alpha$ - flow coefficient

$\rho-\quad$ fluid's density

The demands that need to be satisfied for an improved static and dynamic hydraulic resistances behavior are:

- Constant value of flow coefficient $\alpha$ and as near as possible to 1, as a measure of the hydraulic losses

- Reduced hydrodynamic forces' effect which tend to "close" the resistance; their value is greater the greater the flow is and have an major impact on device performance, but also on the possibility of controlling the fluid flow.

- Reduced cavitation phenomenon that occurs when pressure drops below vapor pressure. In steady flow, if the flow section decreases, the result is an increase of flow velocity and a decrease in pressure. From investigations to date, it appears that a change in the resistances' geometric configuration leads to a reduction of cavitation phenomenon.

CFD type analysis has been developed mainly to visualize complex processes or flows through intricate geometries. It represents a great asset in production process, reducing development costs with the models. A worthy example is that of an, Kim \& Shin [5] that conducted a numerical simulation on several types of control valves with different geometries. Pressure drops, cavitation effect and flow coefficient's variation were analyzed. When compared a conventional valve with a newly designed one, using CFD, they observed an

\footnotetext{
a Florin.Bode@termo.utcluj.ro
} 
improvement in the flow field, with reduced cavitation and better performance in the latter.

Reference [2] presents a comprehensive study on hydraulic resistances with cylindrical slide. It was sought to decrease the Reynolds number as much as possible, thus keeping the flow coefficient at a constant value, also to determine the flow coefficient for different forms of slides, therefore trying to reduce the hydrodynamic forces and also studying the effects of cavitation.

\section{Numerical simulations}

For the CFD simulation a geometrical model (figure 2) was designed in Solid Works that shapes the fluid within a hydraulic resistance with a $0.3 \mathrm{~mm}$ command opening. The fluid, hydraulic oil with $876 \mathrm{~kg} / \mathrm{m}^{3}$ density is pushed in a $15 \mathrm{~mm}$ high and $6 \mathrm{~mm}$ diameter hole with a flow rate of $201 / \mathrm{min}$.

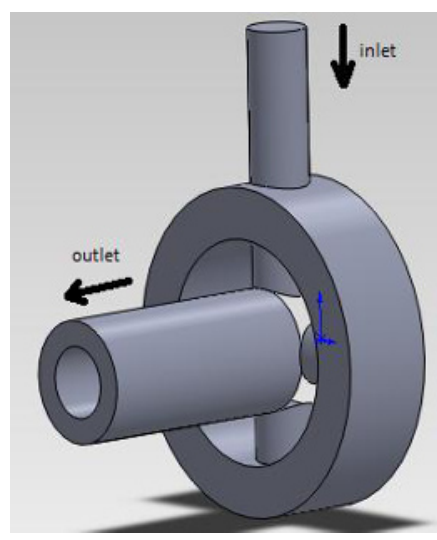

a.

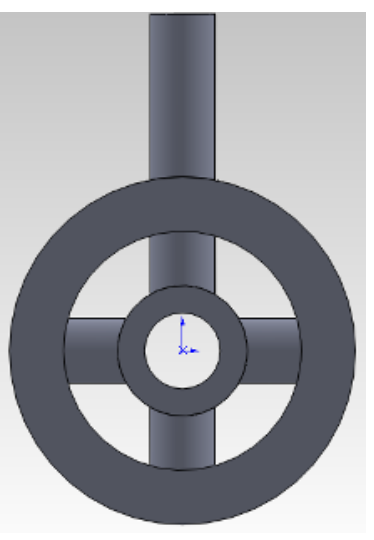

b.
Fig.2 Geometric model

The fluid reaches the spool chamber through four cylindrical orifices (found within the sleeve). Small openings were chosen due to occurrence of hydrodynamic forces, which disturb the dynamic behaviour of the resistance and cavitation phenomenon, resulting from pressure values lower than vapour pressure; this happens due to sudden narrowing of the flow path

The model was imported into Ansys Design Modeller for meshing (figure 3). In order to complete the grid, approximately 2.4 million tetrahedral elements were needed.

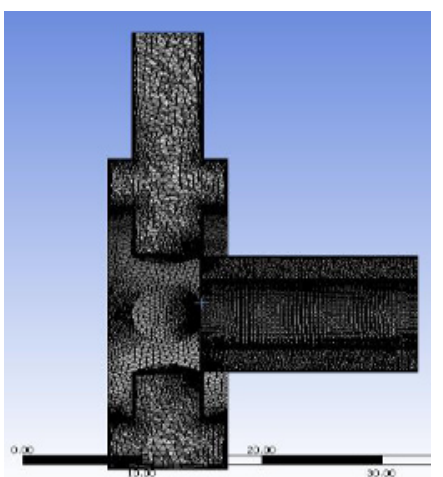

Fig.3 Geometrical mesh
To predict the flow in the command openings, a refined grid was generated, due to high velocity gradients in those areas, becoming sparser toward the boundary layer. Such a discretization detail is shown in figure 4 .

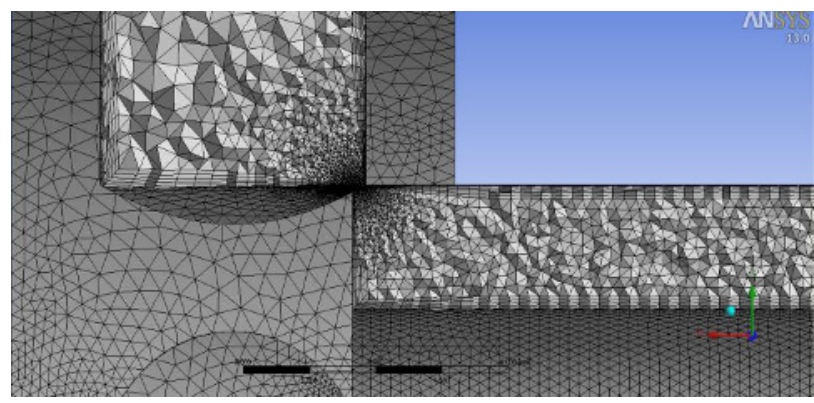

Fig.4 Mesh detail for the command opening

The turbulence model chosen for this application was RNG k- $\varepsilon$. This model derives from the standard $\mathrm{k}-\varepsilon$ turbulence model using a rigorous statistical technique called renormalization group theory. It has a similar form to standard k- $\varepsilon$ turbulence model, but comes with improvements, such as:

The $\varepsilon$ equation has an extra term which significantly increases the working precision

Includes the effect of turbulence vortex, increasing the accuracy of swirling flows

While the standard model uses a high Reynolds number, the RNG theory provides an analytically derived differential equation for viscosity, which is responsible for satisfactory results in small Reynolds numbers.

These features make the RNG k- $\varepsilon$ turbulence model more exact and it can be used on a wider variety of flows than the standard model.

The equations behind this turbulence model are the continuity equation (eq. 2), momentum conservation (eq. 3) and the transport equation (eq. 4):

$$
\begin{gathered}
\frac{\partial u_{x}}{\partial x}+\frac{\partial u_{y}}{\partial y}+\frac{\partial u_{z}}{\partial z}=0 \\
u_{x} \frac{\partial u_{i}}{\partial x}+u_{y} \frac{\partial u_{i}}{\partial y}+u_{z} \frac{\partial u_{i}}{\partial z}=-\frac{1}{\rho}\left(\frac{\partial p}{\partial x_{i}}+\frac{\partial p}{\partial y_{i}}+\frac{\partial p}{\partial z_{i}}\right)+ \\
+v\left(\frac{\partial^{2} u_{i}}{\partial x^{2}}+\frac{\partial^{2} u_{i}}{\partial y^{2}}+\frac{\partial^{2} u_{i}}{\partial z^{2}}\right)
\end{gathered}
$$

where $\rho$ - represents the fluid's density,

$\mathrm{p}$ - fluid's pressure,

$v-$ kinematic viscosity

$$
u_{x} \frac{\partial t}{\partial x}+u_{y} \frac{\partial t}{\partial y}+u_{z} \frac{\partial t}{\partial z}=a\left(\frac{\partial^{2} t}{\partial x^{2}}+\frac{\partial^{2} t}{\partial y^{2}}+\frac{\partial^{2} t}{\partial z^{2}}\right)
$$


For the complete solving of the turbulence model, two other equations are needed, for the turbulent kinetic energy, $\mathrm{k}$ and its dissipation, $\varepsilon$.

$$
\begin{aligned}
& \frac{\partial}{\partial t}(\rho k)+\frac{\partial}{\partial x_{i}}\left(\rho k u_{i}\right)=\frac{\partial}{\partial x_{j}}\left\lfloor\left(\mu+\frac{\mu_{t}}{\sigma_{k}}\right) \frac{\partial k}{\partial x_{j}}\right\rfloor+ \\
& +G_{k}+G_{b}-\rho \varepsilon-Y_{M}+S_{k} \\
& \frac{\partial}{\partial t}(\rho \varepsilon)+\frac{\partial}{\partial x_{i}}\left(\rho \varepsilon u_{i}\right)=\frac{\partial}{\partial x_{j}}\left\lfloor\left(\mu+\frac{\mu_{t}}{\sigma_{\varepsilon}}\right) \frac{\partial \varepsilon}{\partial x_{j}}\right\rfloor+ \\
& +C_{1 \varepsilon} \frac{\varepsilon}{k}\left(G_{k}+C_{3 \varepsilon} G_{b}\right)-C_{2 \varepsilon} \rho \frac{\varepsilon^{2}}{k}+S_{\varepsilon}
\end{aligned}
$$

where $\mathrm{G}_{\mathrm{k}}$ - generation coefficient of turbulent kinetic energy, $S_{k}, S_{\varepsilon}-$ user defined terms, $C_{1 \varepsilon}=1,42, C_{2 \varepsilon}=1,68-$ turbulence model's specific constants [6]

\section{Results and discussions}

Fluent solver gives a choice of several "upwind" interpolation schemes; the one used in this study is "second order upwind" to calculate the convective terms. Pressure-velocity coupling scheme chosen is given by the "simple" algorithm of the solver. Regarding the accuracy of results, the imposed convergence criterion was $10^{-5}$ for all the variables residuals.

As said in first part, this paper deals with a CFD type numerical simulation of flow through a hydraulic resistance with spool and sleeve with four cylindrical orifices.

In order to see the development of the jet flow, in figure 5 can be observed a transversal detail of the mesh used for CFD numerical simulation together with an isosurface of velocity at $120 \mathrm{~m} / \mathrm{s}$.

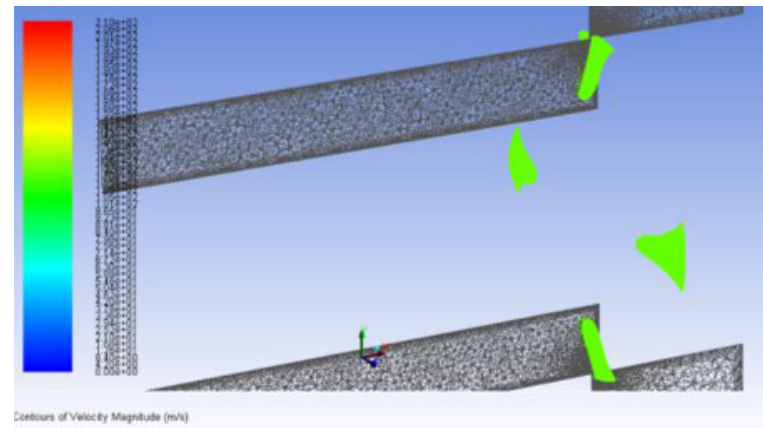

Fig. 5 Isosurface of velocity $120 \mathrm{~m} / \mathrm{s}$

In figure 6 contours of velocity can be observed in the central axial plane near a command opening. The maximum velocity of the flow is $210 \mathrm{~m} / \mathrm{s}$.

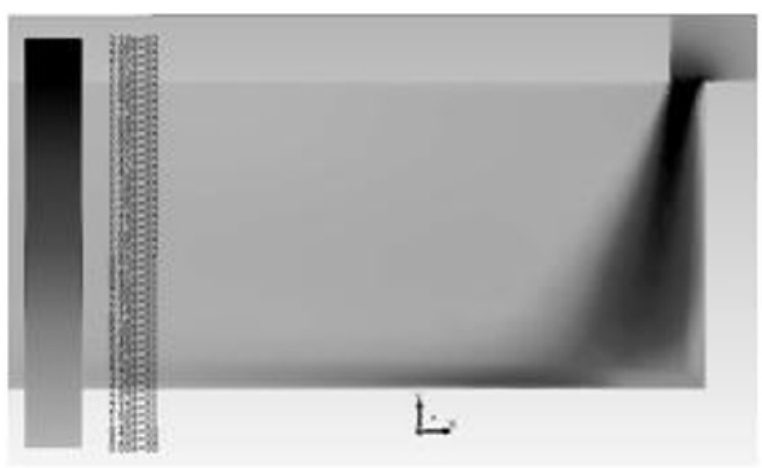

Fig. 6 Contours of Velocity

In the next figure (figure 7) one can see the velocity contours in two different planes, one near the first ridge of command opening (figure 7a) and the second, in the middle of the opening (figure $7 \mathrm{~b}$ ).

a

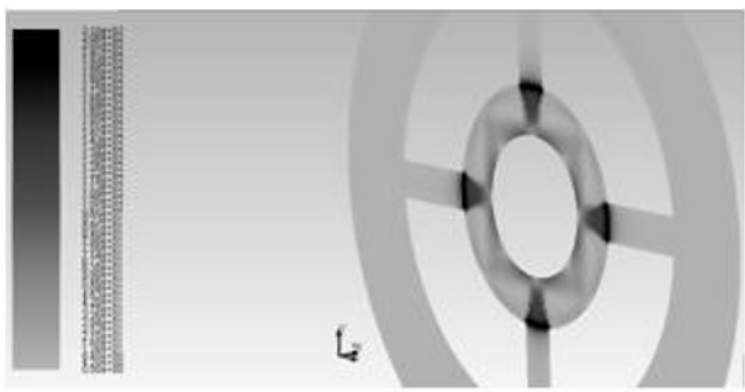

b

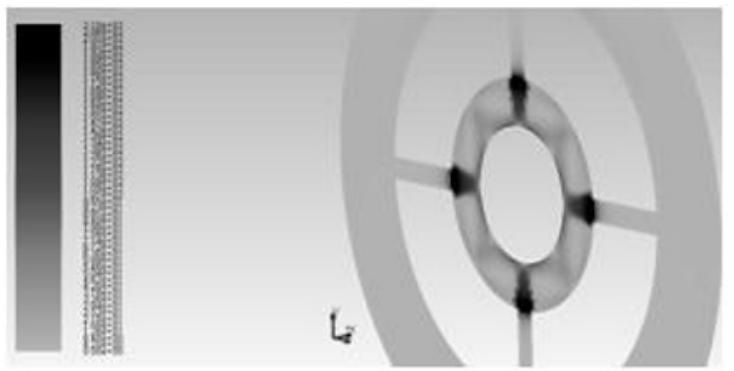

Fig. 7 Velocity profile in yx plane a)near the first ridge of the command opening; b) in mid - section of the command opening

In figure 8 the pressure contour can be observed (figure 8a) with a detail representing where the cavitation phenomenon may occur (figure $8 \mathrm{~b}$ ) .This is due, as said before, to the sudden decrease of the flow section and the increase of velocity in that area. Figure 9 presents the same pressure profile, but in a yz plane positioned in the middle of the opening along a transversal detail of the mesh. 


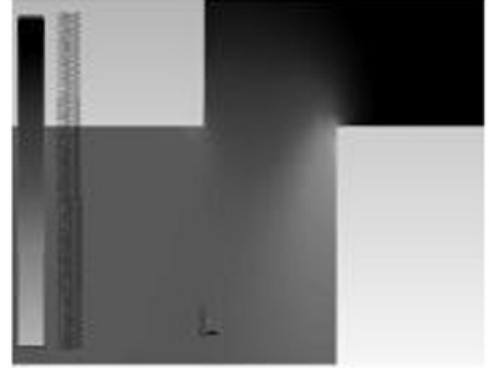

a)

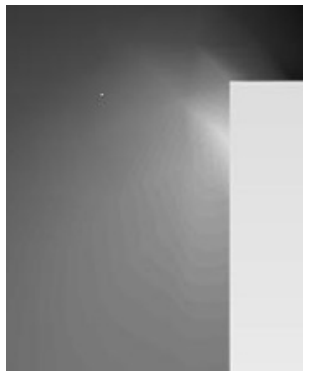

b)
Fig. 8 Contours of pressure a).command opening area b).detail with the cavitation zone

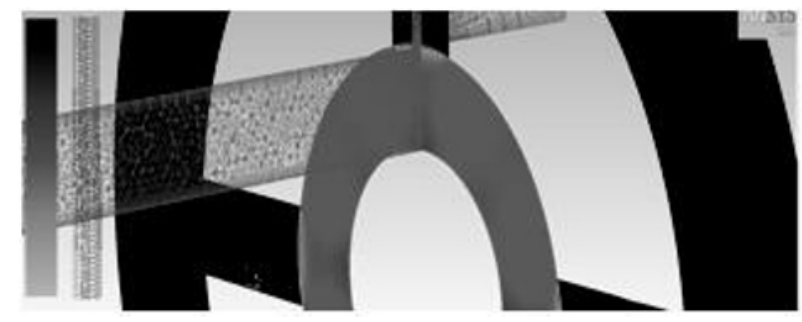

Fig. 9 Contours of pressure

The production of turbulent kinetic energy is more intense in the opening as seen in figure 10. Turbulent kinetic energy production is generated first by the shoulder of the opening and second due to vortices (figure 11).

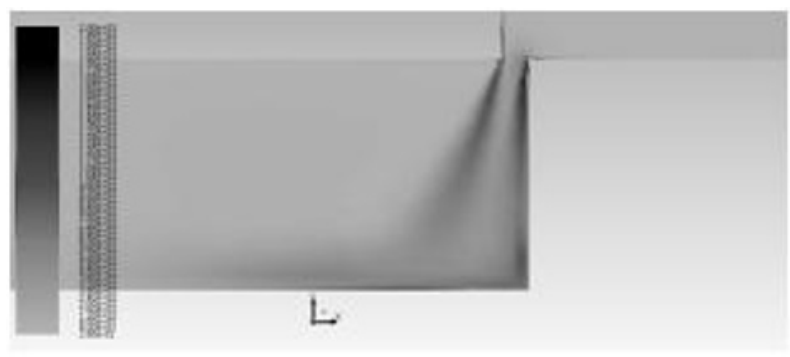

Fig. 10 Contours of turbulent kinetic energy

It can be seen how the flow detaches from the wall when it hits the first ridge and further downstream reattaches to it (Figure 11). This "bubble" (recirculation area) is effectively narrowing the flow section, causing increased velocity, thus decreasing the pressure. $[2,7]$

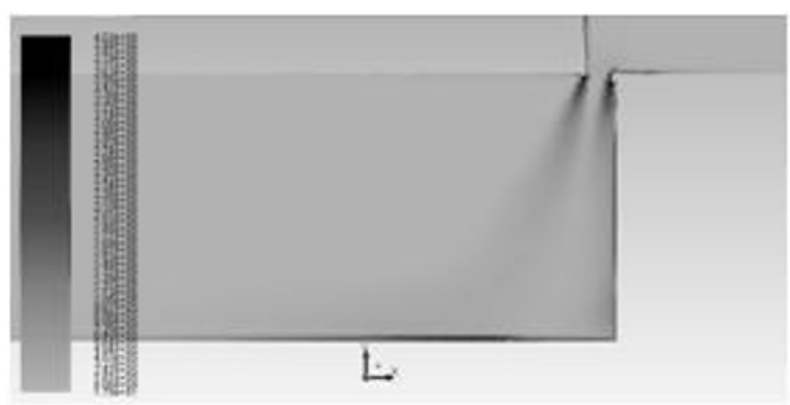

\section{Conclusions}

This paper presented a CFD numerical simulation of flow through a hydraulic resistance with spool and sleeve with four cylindrical orifices. The resistance's command opening was $0.3 \mathrm{~mm}$. The RNG k- $\varepsilon$ turbulence model was used to simulate this flow and velocity and pressure fields were observed. Therefore, the maximum speed registered was $210 \mathrm{~m} / \mathrm{s}$; this generated a pressure drop in the studied section that may lead to cavitation occurrence.

Comparisons to authors' recent studies on this matter, mainly on the same type of hydraulic resistance but with a command opening of $0.1 \mathrm{~mm}$, reveal that the velocity value in the critical section is five times lower in the $0.3 \mathrm{~mm}$ opening, with the same boundary conditions (flow rate $201 / \mathrm{min}$ and $6 \mathrm{~mm}$ diameter of the orifices).

Furthermore investigation will be made, with other geometrical patterns for the sleeve's orifices and the one that will generate the optimal results will be executed for experimental investigations.

ACKNOWLEDGMENT: This paper was supported by the project "Improvement of the doctoral studies quality in engineering science for development of the knowledge based society-QDOC" contract no. POSDRU/107/1.5/S/78534, project co-funded by the European Social Fund through the Sectorial Operational Program Human Resources 2007-2013.

\section{References}

1 T. Canta, IPCN, 1982

2 D. Opruţa, Cluj-Napoca, PhD Thesis, 1998

3 W. Backe, ,Servohydraulik”, RWTH, Aachen, 1984

4 I. Deacu, D. Banabic ş.a. - Ed. Dacia, Cluj-Napoca, 1989

5 Y. J. An, B. J. Kim, B. R. Shin, Journal of Mechanical Science and Tehnology 22, 2008; 19982005

6 Ansys 12.0 Theory Guide

7 J. M. McDonough, Universityof Kentucky, Lexington, 2009

Fig. 11 Contours of vorticity 